FoGG, G. E. (1957). J. gen. Microbiol. 16, 294-297

\title{
Relationships between Metabolism and Growth in Plankton Algae
}

\author{
By G. E. FOGG \\ Department of Botany, University College London
}

As an organism grows the pattern of its metabolism changes and, except under special conditions of culture, even the average chemical composition or activity of a population does not remain constant with time. Knowledge of the relationships between growth and metabolism in plankton algae is clearly worth having since the nature and intensity of the metabolic activities of these organisms in nature cannot generally be determined directly, but must be inferred from determinations of population densities and the chemical and physical properties of the waters in which they have developed.

Few truly planktonic algae have been isolated in pure culture and laboratory investigations on these have scarcely begun. For the present, therefore, we are forced to use information derived largely from studies with non-planktonic algae in interpreting the behaviour of plankton. It cannot be assumed, of course, that planktonic algae always behave in a similar manner to the sewage alga Chlorella for example, but the now quite substantial body of information about the metabolism of this and a few other algae is beginning to give a picture which is probably of general application. It is my purpose in this paper to sketch the outlines of this picture and to discuss some of its applications to plankton.

When an alga is inoculated into a limited amount of culture medium and exposed to suitable conditions of light, temperature and aeration, it shows more or less well-defined phases of growth following each other in the familiar pattern, i.e. there is a lag phase, an exponential phase, a phase of declining relative growth rate and a stationary phase. Few studies have been made on the lag phase in algae but it has been found in some species that the cell protein and nucleic acid contents increase and we may surmise that this phase is one of reconstitution, in which enzyme and substrate concentrations are built up to the degrees necessary for multiplication. In the exponential phase the organisms have a high capacity for photosynthesis and the products are used mainly for the synthesis of protein. In a continuous culture, in which the population density is kept constant by the regulated addition of fresh medium, this phase of growth can be maintained indefinitely with the chemical composition and physiological attributes of the organisms remaining constant. In a culture of limited volume, however, the exponential phase ends after a time because of depletion of nutrients, accumulation of toxic by-products of metabolism, or simply because light becomes limiting as the culture becomes more dense.

By the end of the exponential phase the capacity of the organisms for 
photosynthesis has declined considerably and its products become increasingly diverted along pathways other than that of protein synthesis to form 'reserves' of fat or carbohydrate. These changes are not abrupt but gradual, beginning well before exponential increase in number of organisms ceases. Thus the decline in photosynthetic capacity begins a day or two before that of relative increase in numbers of organisms. The plotting on a logarithmic grid of the total amounts of various fractions in cultures as a function of dry weight generally yields straight lines, showing that the differential rates of increase of the different components remain constant over almost the whole period of culture. It seems that constant relative increase in number of organisms in such cultures does not represent a steady state condition but that it may be maintained while considerable changes of cell composition in terms of major fractions such as protein, fat and carbohydrate are taking place. The direction of these changes depends on culture conditions; we have a great deal to learn about this still but it seems generally true that when nitrogen is limiting the products of photosynthesis are used by algae for fat synthesis, whereas when phosphate is in short supply carbohydrate is accumulated rather than fat.

There is increasing evidence that extracellular products of algae are often important quantitatively and also because of their biological effects. All algae which have been examined from this point of view have been found to liberate peptides into the medium during the course of growth (Fogg \& Westlake, 1955). The relative amount of these substances is increased by deficiencies of certain elements and can account for as much as $50 \%$ of the total nitrogen assimilated, but their liberation seems to be invariably linked with growth and to be independent of the volume of the medium. Organic acids are also prominent among the extracellular products of at least some species. Glycollic acid, for example, has been found to be liberated in substantial amounts from photosynthesizing Chlorella (Tolbert, 1955) and this and other organic acids have been found to accumulate in amounts corresponding to $10-45 \%$ of the total organic material formed in cultures of various Chlamydomonas spp. (Allen, 1956). It is to be expected that, with metabolites of low molecular weight like organic acids, there is an equilibrium between the intra- and extracellular concentrations so that the relative amount liberated from within the organisms should be dependent on the volume of the medium. Such a relationship, however, does not appear to have been demonstrated experimentally. Evidence is accumulating to show that many algae produce antibiotic extracellular substances (Lefèvre, Jakob \& Nisbet, 1952; Steemann Nielsen, 1955), but the chemical nature of these and the physiological relations in their production have yet to be established.

Some of the applications of these findings to plankton may now ke considered. A phase of protoplasnic growth without cell division, resembling the lag phase in cultures, is probably necessary before populations of plankton algae begin to increase but, on the face of it, appears difficult to study directly. Possibly such a lag phase is responsible for the phenomenon of the 'spring outburst'. It is well known that many micro-organisms cannot begin exponential growth until particular concentrations of certain metabolites have been 
established in the external medium. Were a similar state of affairs to exist for phytoplankton increase of numbers would not necessarily occur under favourable conditions of light, temperature and nutrient supply, but would have to await the establishment of the requisite concentration of metabolites in the water immediately surrounding the organisms. This explanation is consistent with the observed correlation between the spring outburst and decreasing turbulence.

Something resembling exponential growth occurs in natural phytoplankton populations, but whether or not the same sort of variation in metabolic pattern and activity of the organisms occurs as in laboratory cultures remains to be demonstrated. A phase of intense photosynthetic activity similar to that observed at the beginning of the exponential phase in culture may be difficult to detect on account of its transient nature and the low population density. Recorded maximum rates of photosynthesis of natural phytoplankton populations evidently all represent later stages of growth since they are nearly all of about the same magnitude and about one-third of those recorded for actively growing laboratory cultures of Chlorella when expressed on the basis of oxygen evolution per unit volume of cell material or per unit weight of chlorophyll (Verduin, 1952; Ryther, 1956). In the later stages of exponential growth and in the phase of declining relative growth, such as are most usually encountered in phytoplankton populations, the products of photosynthesis may be expected to be used mainly in synthesis of 'reserve' products. In phytoplankton these are usually fats and their synthesis would give a photosynthetic quotient of the order of $\mathbf{1 . 4}$. Thus if a value of unity be assumed for the photosynthetic quotient, as is usual in studies on plankton productivity, the amount of carbon fixed as calculated from observed oxygen evolution may be considerably overestimated. Ryther (1956) has considered this problem and has concluded that a value of $\mathbf{1 . 2 5}$ for the photosynthetic quotient would be more realistic.

There are, of course, important differences between the growth of natural phytoplankton populations and that of algae in laboratory cultures of limited volume. Interaction with other organisms may result in considerable modification of growth and metabolism. Zooplankton decreases phytoplankton numbers by grazing but also, together with bacteria, continuously liberate plant nutrients so that phytoplankton multiplication takes place even though there are no detectable amounts of these nutrients in the water. On the other hand, staling products which would accumulate in pure cultures may be removed or otherwise inactivated by associated organisms. Thus a natural phytoplankton population which is stationary as far as numbers/unit volume is concerned is not likely to be physiologically equivalent to a stationary population in laboratory culture. Moreover, a natural population is not limited to a definite volume of water but can expand into the surrounding water. In that it may have a continuous supply of nutrients whilst its staling products are continually removed or diluted, a natural phytoplankton population perhaps resembles in its physiological condition that in continuous culture more closely than that in a limited volume of medium. 
Extracellular products of plankton are of interest in several ways, but mention can only be made here of their possible significance in relation to productivity studies. If there is indeed a tendency towards equilibrium between intra- and extracellular concentrations of certain metabolites then it might be that the loss of such substances from the organisms at low plankton densities might be relatively great so that methods such as the radiocarbon method of Steemann Nielsen, which take account only of carbon fixation in particulate matter, may seriously underestimate the total productivity. This might account for the discrepancy between the results given by the Steemann Nielsen method on the one hand and the Gaarder and Gran method on the other, for oligotrophic waters. Preliminary studies with radiocarbon as a tracer have shown that the amount of photosynthetically-fixed carbon in filtrates of a eutrophic lake water is only about $0.5-1 \%$ of that in the plankton retained on the membrane filter and that this proportion does not increase as the plankton concentration is decreased. However, we know too little about the physiology of the extracellular products of algae to be sure that a similar relation holds for different physiological states and for all types of plankton.

\section{REFERENCES}

Aluen, M. B. (1956). Excretion of organic compounds by Chlamydomonas. Arch. Mikrobiol. 24, 163.

FogG, G. E. \& Westlake, D. F. (1955). The importance of extracellular products of algae in freshwater. Verh. int. Ver. Limnol. 12, 219.

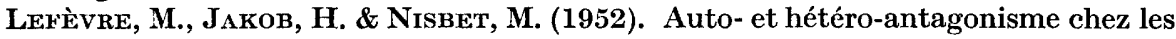
algues d'eau douce in vitro et dans les collections d'eau naturelles. Ann. Sta. cent. Hydrobiol. appl. 4, 5.

Niersen, E. S. (1955). The production of antibiotics by plankton algae and its effect upon bacterial activities in the sea. Papers in Marine Biology and Oceanography, p. 281. London: Pergamon Press Ltd.

Ryther, J. H. (1956). The measurement of primary production. Limnology and Oceanography, $1,71$.

Tolbert, N. E. (1955). Excretion of glycolic acid by algae during photosynthesis. Fed. Proc. 14, part I, p. 292.

Verduin, J. (1952). The volume-based photosynthetic rates of aquatic plants. Amer. J. Bot. 39, 157. 